\title{
Representaciones Sociales de la Inteligencia
}

\author{
Elena Zubieta y J ose Valencia*
}

\section{Resumen:}

El presente artículo indaga en la relación entre teorías explícitas e implícitas de la Inteligencia. En base a la investigación de Mugny \& Carugati (1989) se diseñó un cuestionario auto-administrado que respondieron 286 sujetos. Los resultados encontrados son congruentes con las principales hipótesis postuladas: Es posible hablar de un solapamiento general entre las teorías explícitas e implícitas. Similar a lo que ocurre actualmente en el discurso de los expertos, en las respuestas de los sujetos la Inteligencia aparece como un atributo relacionado con la adaptación a las normas sociales y variables contextuales son importantes a la hora de definirla. Sin embargo, concepciones basadas en la "ideología del dotado" tienen aún una fuerte presencia, lo que confirma que determinismo biológico y determinismo sociológico son los principales ejes alrededor de los cuales se organizan las argumentaciones. Del mismo modo, la familiaridad y la identidad social se refuerzan como principios organizadores de una representación social. La distancia con el objeto, medida como la creencia en las diferencias de inteligencias como un problema sin o con solución, y el nivel de implicación con la problemática, maestros y no maestros, se observan como elementos discriminantes al momento de apoyar dimensiones específicas.

Palabras Claves: Representaciones Sociales, Inteligencia, Teorías Implícitas, Sentido Común.

\section{Abstract:}

\section{Social Representations of Intelligence}

The article stresses the relationship between Explicit and Implicit theories of Intelligence. Following the line of common sense epistemology and the theory of Social Representations, a study was carried out in order to analyze naive's explanations about Intelligence Definitions. Based on Mugny \& Carugati (1989) research, a self-administered questionnaire was designed and filled in by 286 subjects. Results are congruent with the main hyphotesis postulated: A general overlap between explicit and implicit theories

* Facultad de Psicología. Universidad del País Vasco. Avenida de Tolosa 70. 20018 San Sebastián. España. E-mail: psazucae@sc.ehu.es 
Psicodebate. Psicología, Cultura y Sociedad

showed up. According to the results Intelligence appears as both, a social attribute related to social adaptation and as a concept defined in relation with contextual variables similar to expert's current discourses. Nevertheless, conceptions based on "gifted ideology" still are present stressing the main axes of Intelligence debate: biological and sociological determinism. In the same sense, unfamiliarity and social identity are reaffirmed as organizing principles of social representation. The distance with the object -measured as the belief in intelligence differences as a solve/non solve problem- and the level of implication with the topic -teachers/no teachers- appear as discriminating elements at the moment of supporting specific dimensions.

Key words: Social Representations, Intelligence, Implicit Theories, Common Sense. 


\section{Introducción}

En los últimos veinte años se ha podido observar, en el área de la psicología social, un creciente interés por los estudios sobre lo que se denominó conocimiento «ingenuo» o «de sentido común». La necesidad de entender los esquemas de acción y la organización del conocimiento en los individuos llevó a las «perspectivas ingenuas» al centro de la discusión y el análisis de una importante parte de la psicología sociocognitiva. La bifurcación entre un pensamiento conforme a la ciencia y la razón y la realidad del pensamiento en el mundo social aparecían a través de diferentes etiquetas: lógica y mito; pensamiento doméstico y pensamiento salvaje (Lévi-Strauss, 1962); mentalidad lógica y mentalidad psicológica (Lévy Bruhl, 1922); pensamiento crítico y pensamiento automático (Moscovici, 1981). Estas etiquetas no describían sino la divergencia entre dos modos de conocimientos o adquisición de conocimientos: uno normalizado y otro no normalizado (Moscovici \& Hewstone, 1988).

De esta manera, surge una epistemología del sentido común que opone al pensamiento normalizado, que reflexiona, se controla y formula criterios para invalidar o confirmar sus razonamientos, un pensamiento no normalizado que se corresponde con un pensamiento más natural, más innato y que se adquiere sin una formación particular. Moscovici \& Hewstone (1988) sostienen que la explicación de las diferencias entre estos pensamientos es básicamente la tarea que la teoría de las Representaciones Sociales se propone enriquecer, en sus palabras: «Una epistemología popular tiene por objeto estudiar un tipo determinado de conocimiento, a saber, el sentido común» (pag. 691).

Lo que subyace a esta línea de abordaje es la relación ciencia-cotidianeidad y las características peculiares que ésta adquiere en la sociedad moderna. Durante el transcurso del siglo XIX un interesante cambio se da entre ciencia y sentido común, contrariamente a lo que se pensaba en el siglo anterior, lejos de ser el antídoto de representaciones e ideologías, la ciencia es, en realidad, su fuente. El mundo de lo científico se encuentra tan alejado de lo cotidiano que resulta totalmente extraño para el profano quien sólo puede tener acceso a ese mundo si sus conceptos claves, expresados en símbolos y ecuaciones matemáticas, son traducidos a términos familiares. De este modo se le puede estimular a que piense en los átomos como si fueran bolas de billar (Farr, 1987).

Esta relación es particularmente interesante por dos motivos fundamentales: en primer lugar por sacar a la luz un conocimiento práctico que se constituye a partir de las experiencias, de las informaciones, conocimientos y modelos de pensamiento que recibimos y trasmitimos a través de la tradición, la educación y la comunicación social. En segundo lugar, por sostener que las teorías en las ciencias sociales representan elaboraciones del lenguaje cotidiano, y que es un error de los científicos sociales creer que pueden tener una perspectiva acerca de la vida social que trasciende los límites del sentido común compartido por una cultura.

En este sentido, es una práctica casi cotidiana hacer que lo extraño resulte familiar y lo invisible, perceptible. La amenaza que conlleva lo desconocido o insólito y la falta de 
una categoría en la cual clasificarlo genera la aparición de representaciones sociales en la comunicación humana : «resulta evidente que éstas trascienden la esfera de las simples opiniones, imágenes y actitudes...se trata de sistemas cognitivos que poseen una lógica y un lenguaje particulares, de «teorías», de «ciencias» sui generis, destinadas a descubrir la realidad y ordenarla» (Moscovici \& Hewstone, 1988; pag. 692).

Ciencia y cotidianeidad se retroalimentan y se entremezclan para dar sentido a la dinámica social permitiendo a los individuos orientarse en su entorno social y material, y dominarlo.

Semin (1987) sostiene que el solapamiento entre modelos ordinarios de lenguaje de realidades psicosociales y varios modelos científicos indica que las estructuras científicas son abstracciones idealizadas y que ambas, son las precondiciones y consecuencias de las referencias a temas como la inteligencia, la adicción a drogas o el desarrollo en el discurso cotidiano. Así, la investigación científica en temas tales como el aprendizaje o el desarrollo toman como punto de partida el edificio conceptual de entendimiento de sentido común de la inteligencia y particularmente de los orígenes de las diferencias interindividuales, un conocimiento detallado de la forma en que la gente de una cultura específica piensa acerca de este fenómeno. Sin este prerequisito parece imposible para un investigador usar, por ejemplo, el término «inteligencia» de una manera culturalmente sensible.

En el área de los estudios sobre Inteligencia, tema que nos ocupa, Carugati (1990) cree que el solapamiento entre teorías implícitas y explícitas no debe sorprendernos, es su ausencia la que resultaría extraña. Los expertos, al igual que las personas «ingenuas», son participantes de la construcción simbólica de la realidad, unos como otros se informan en base a las teorías implícitas elaboradas en este proceso. Lo importante para el autor son las implicaciones que estas consideraciones conllevan pues implican que los análisis empíricos en psicología social presuponen un entendimiento de sentido común del mundo social que los investigadores comparten con sus sujetos de análisis.

En la investigación empírica son muchos y variados los trabajos que tratan de indagar acerca del problema de la inteligencia, su definición y desarrollo, sin embargo, todavía son pocos los trabajos que incluyen a las construcciones que los individuos elaboran para explicar las diferencias de inteligencias que existen entre los individuos y, lo que es más interesante aún, que indaguen en la relación que aquellas tienen con las teorías científicas.

Dentro de la producción y comunicación científica nos encontramos con una multiplicidad de definiciones de inteligencia, la diversidad de visiones parece ser la característica de la temática pero, como bien expresan Mugny \& Doise (1985), esto no impide la construccción de representaciones sociales de la inteligencia. La necesidad de explicar las diferencias de rendimiento y capacidades de los individuos inicia un proceso de generación de una representación, pone en funcionamiento un proceso simbólico de afrontamiento e integración en el sentido común. En este sentido, y como afirma Carugati (1990), todas las personas tenemos una teoría de la inteligencia y, más allá de la diversidad de las definiciones se puede hablar de una suerte de consenso. Los intentos por explicar las diferencias de capacidad mental aluden al viejo debate naturaleza- 
medio que ha sido usado para justificar un continuo reforzamiento de una psicología particular o de la «ideología del dotado». Una explicación más completa se lograría, a través de indagar en las teorías implícitas, si tenemos en consideración el amplio rango de elementos que en el proceso conectan al niño, al adulto y al desafío cognitivo.

En este sentido, parece necesario estar prevenidos de las formas a través de las cuales los adultos y estudiantes categorizan a la inteligencia y el desarrollo intelectual para comprender la dinámica sociocognitiva que se establece entre ellos. Dentro de los sujetos involucrados en esta interacción, los autores enfatizan el vacío de conocimiento que hay respecto de «las representaciones sociales que se generan entre los adultos quienes desde sus lugares de maestros, padres u otro rol: I) participan en iniciar y moldear las interacciones sociales con los niños; II) están implicados en la interpretación y creación de las representaciones sobre las situaciones sociocognitivas con las cuales se confrontan constantemente y, III) como parte del proceso, crean estrategias para ayudar al niño» (Mugny \& Doise, 1985)

Es el conflicto sociocognitivo que las diferencias de inteligencias entre los individuos provoca y su resolución en tanto sistemas de argumentación y explicación, el que intentamos analizar en diferentes partes y niveles. Orientados desde las explicaciones "científicas", indagamos en las explicaciones de sentido común de actores con distintos niveles de implicación respecto de la temática. En la articulación entre teorías explícitas e implícitas está la fuerza que lleva a interesarnos por el análisis de la sociogénesis y la ontogénesis de la realidad simbólica, estudiando la interacción de lo individual y lo colectivo (Carugati, 1990).

\section{¿Por qué la Teoría de las Representaciones Sociales?}

Desde el punto de vista de los abordajes sobre el desarrollo cognitivo, Mugny \& Doise (1985) sostienen que, teóricamente, éstos pueden describirse a través de los nombres de Piaget y Bourdieu. Mientras la línea piagetiana trata el tema de la inteligencia y su desarrollo desde una perspectiva individual, los trabajos de Bourdieu enfatizan las determinaciones sociales de la inteligencia, institucionalizada básicamente a través del éxito escolar. Ambas teorías crecieron independientemente mostrando la ausencia de una visión articulada del desarrollo cognitivo. A través de la teoría de las representaciones sociales podemos encontrar un espacio de interacción entre la Sociología y la Psicología. El concepto desarrollado por Moscovici (1961) puede verse como proveyendo al desarrollo representacional de Piaget de la ausente dimensión social de la misma manera que, enriquece con elementos sociocognitivos al concepto de habitus de Bourdieu.

La teoría de las RS (Moscovici, 1961) no es el único marco conceptual que estudia las creencias compartidas pero sí constituye una nueva reorientación cuyos principales elementos incluyen una metateoría social construccionista. Provee de un marco novedoso que desafía a la psicología social a interesarse por aquellas explicaciones y razonamientos o visiones del mundo, mantenidas y compartidas por un gran número de personas. 
También se reconoce a esta teoría por el resurgimiento que hace de los componentes sociales del pensamiento largamente olvidados por los estudios de las actitudes y la cognición. Fraser (1994) indica que los estudios sobre RS han sido más valiosos que aquellos sobre actitudes, al establecer que las creencias ampliamente distribuidas (widespread beliefs) se externalizan e institucionalizan. Asimismo, Agoustinnos \& Innes (1990) establecen que las RS y la teoría que las sustenta, tienen el potencial de reintroducir una mirada social y colectiva a la investigación psicosocial al proveer de una amplia dimensión social a los abordajes de la cognición social.

De acuerdo con Moscovici (1984), una Representación Social es "la elaboración que de un objeto social realiza una comunidad o grupo social con el propósito de comunicarse y actuar»; la información no sólo depende de las interacciones entre los miembros del grupo y las instituciones, sino también de operaciones de mecanismos psicológicos básicos, particularmente la objetivación y el anclaje que sirven para transformar lo no familiar en familiar, para hacer las cosas "más comprensibles" para los sujetos

Las RS no están libres de valores, sino que poseen una función prescriptiva con implicaciones de acción o conducta. Son, simultáneamente, conjuntos consensuados de cogniciones y evaluaciones, es decir, sistemas de valores, ideas y prácticas en constante movimiento y cambio. No son necesariamente hegemónicas, en dominios particulares, puede haber RS alternativas. En estos téminos de consenso, las RS pueden tomar diferentes formas: "hegemónicas", “emancipadas" y "polémicas", lo que implica, como Moscovici (1984) postula, que el pensamiento del grupo nunca es algo fuertemente consensuado ni tampoco una conglomeración de ideas idiosincráticas mantenidas por individuos aislados.

A pesar de que la teoría ha sido, y aún es, foco de diferentes críticas, (sobre todo por la ambigüedad que se sostiene tiene el concepto central) es un marco teórico-metodológico rico para describir y evaluar las teorías implícitas que los sujetos tienen respecto de un determinado objeto social, en nuestro caso, los razonamientos y explicaciones que las personas construyen a partir de la diferencia de inteligencia en los individuos.

\section{Las concepciones «científicas» de la Inteligencia:}

Dentro de la perspectiva sociocognitiva la Inteligencia puede ser definida, de manera amplia, como la capacidad para adaptarse al ambiente y de aprender de la experiencia (Sternberg \& Kaufman, 1998).

Carugati (1990) refiere a una colección editada por Fry (1984) en la que aparecen reseñados los cambios producidos en las concepciones científicas de la inteligencia. A ellas subyacen como temas de discusión: I) Una visión contextualista. La inteligencia aparece como una compleja mezcla de ingredientes que pueden diferir de un medio socio-cultural a otro; II) Un relativismo cultural. La inteligencia se define según diferentes visiones del mundo; III) Una conceptualización ecologista que enfatiza las relaciones dinámicas entre el sujeto y el ambiente. Los sujetos están orientados a la resolución de problemas y a actividades direccionadas según sus objetivos bajo el marco ecológico 
de sus culturas y dentro de la cultura como un todo; IV) El impacto y evaluación de la teoría de los estadíos de Piaget en el desarrollo cognitivo, particularmente en la psicología occidental y; V) Un cambio en la conceptualización de la inteligencia como una cualidad que las personas poseen en diferentes grados. Se incluyen aspectos relacionados al proceso de opinión o emisión de juicios y las reglas y protocolos que subyacen al despliegue del comportamiento inteligente.

En una revisión de Sternberg \& Kaufman (1998) de las denominadas teorías amplias de la inteligencia y de los tipos de inteligencia encontramos:

I) la teoría de las Inteligencias Múltiples. Gardner (1983) propone que lo que existe no es una inteligencia única sino un conjunto de inteligencias relativamente distintas, independientes y múltiples: a) Lingüística (usada para leer libros o escribir poemas); b) Lógico-matemática (usada para derivar pruebas lógicas o para resolver problemas matemáticos);c) Espacial (usada para acomodar maletas en el maletero de un coche); d) Musical (usada para cantar una canción o componer una sinfonía); e) Corporal Kinestética (usada para bailar o jugar al futbol); f) Interpersonal (usada en entender e interactuar con otras personas) y; g) Intrapersonal (usada para entenderse a uno mismo). Recientemente Gardner (1998) ha incorporado la inteligencia naturalista como aquella que describe personas capaces de discernir dinámicas o esquemas en la naturaleza.

II) la teoría de la Inteligencia Verdadera. Perkins (1995) indica tres aspectos básicos en ella: neuronal, experencial y reflexiva. La Inteligencia neuronal es el funcionamiento de los sistemas neuronales de las personas, algunos funcionan más rápido y con mayor precisión que otros y es «imposible de enseñar» (similar a la inteligencia fluida de Cattell). La inteligencia experencial es la que se aprende de la experiencia. Es la medida y organización del conocimiento de base (inteligencia cristalizada de Cattell). El aspecto reflexivo refiere al rol de las estrategias en memoria y solución de problemas, similar a la metacognición o monitoreo cognitivo.

III) Modelo Bioecológico de Inteligencia. Ceci (1996) propone este modelo según el cual múltiples potencialidades cognitivas, de contexto y conocimiento, son bases esenciales en el rendimiento diferencial de las personas. Es difícil si no imposible separar claramente las contribuciones bilógicas y ambientales a la inteligencia. Las habilidades pueden expresarse de muy diferente manera en contextos diferentes.

IV) Inteligencia Emocional. Es la habilidad para percibir adecuadamente, evaluar y expresar las emociones; para acceder y/o generar sentimientos cuando facilitan el pensamiento; la habilidad para entender las emociones y el conocimiento emocional; para regular las emociones con el fin de promover crecimiento emocional e intelectual. El concepto fue introducido por Salovey \& Mayer $(1990,1993,1997)$ y popularizado y expandido por Goleman (1995).

V) El propio Sternberg (1985) extrae de una variedad de definiciones tres tipos principales de Inteligencia: "habilidad para resolver problemas", "habilidad verbal" y "competencia social". En un trabajo más reciente Sternberg (1996) sugiere prestar menos atención a las nociones convencionales y propone la "inteligencia exitosa" definiéndola como «la habilidad para adaptarse, moldearse, y seleccionar ambientes adecuados para 
nuestros objetivos y los objetivos de nuestra sociedad y cultura». Esta inteligencia involucra un discernimiento individual de las fuerzas y debilidades, y luego la capacidad de ver las formas de capitalizar esas fuerzas y compensar las debilidades. Tres habilidades son necesarias: análiticas, creativas y prácticas. Las analíticas se requieren para analizar y evaluar las opciones disponibles para uno en la vida. Las creativas son necesarias para generar opciones de problema-solución. Los individuos creativos son aquellos que «compran bajo y venden alto» en el mundo de las ideas. Se ha demostrado que estas habilidades son parcialmente distintas a las convencionales del CI y que responden moderadamente a dominios específicos, es decir, la creatividad en un dominio (como el arte) no necesariamente implica creatividad en otro (como la escritura) (Sternberg \& Lupart,1995).

Carugati (1998) compara dos simposias realizados sobre la Inteligencia, uno en 1921 y otro en 1986. En las definiciones propuestas por los expertos 66 años después se observa un solapamiento moderado, atributos tales como adaptación al medio y pensamiento de alto orden (razonamiento y resolución de problemas) destacan en ambos eventos. Del mismo modo, la cuestión sobre inteligencia simple o múltiples continúa siendo un eje importante de discusión aunque no se logra consenso. Los expertos acuerdan y desacuerdan en varias dimensiones: clásicas, dilemáticas, y a través de décadas; simples o múltiples, individual vs. cultural.

\section{Los expertos y el conocimiento «ingenuo» de la Inteligencia:}

Como dijímos al inicio de este trabajo, en las últimas dos décadas un creciente interés por las construcciones no «normativizadas» del conocimiento ha tomado creciente interés. En lo que respecta a la problemática de la Inteligencia, podemos citar trabajos tales como los de Flament (1987), Mugny \& Doise (1985) Sternberg y colaboradores (1981), entre otros. Utilizando el marco teórico de las representaciones sociales, ha sido la investigación de Carugati \& Mugny (1989) la más renombrada. Actualmente se puede apreciar un aumento considerable de las investigaciones que abordan el tema de la Inteligencia y el desarrollo cognitivo de la mano del concepto creado por Moscovici (Poeschl \& Doise, 1993; Doise \& Mugny, 1997; Faria \& Fontaine, 1994, Amaral, 1997).

Sternberg y sus colaboradores $(1981,1995)$, encontraron que las concepciones «ingenuas», no estrictamente iguales, mostraban una similitud general a aquellas mantenidas por los expertos, lo cual refuerza la posibilidad de que las concepciones «populares» puedan constituir una de las principales bases de las teorizaciones científicas, donde aquellas se sistematizan y legitiman.

Asimismo, tema que luego retomarán Mugny \& Carugati (1989), se observó que aunque los modos de hablar sobre una definición de la inteligencia presentarán cierta diversidad, el término mismo no parece cuestionarse, el énfasis en el discurso sobre la inteligencia puede variar pero aún así es «algo» a lo que la gran mayoría acuerda en otorgar un valor positivo, es un valor social de primera importancia. Describir a una persona como inteligente no sólo nos permite evaluarla y clasificarla sino que también 
tiene un rol fundamental en la posición social (y los privilegios a ésta asociados) a la que la persona puede desear y acceder, así, las concepciones de la inteligencia guardan profunda relación con la identidad social.

En 1989 aparece el libro de Mugny \& Carugati "Social Representations of Intelligence" que describe la investigación deasarrollada por ellos a fin de indagar la forma y contenido de las explicaciones sobre la inteligencia de sujetos de diferentes posiciones: Maestros, Padres, Estudiantes y Madres trabajando dentro y fuera del hogar. Los autores llegan a cuatro conclusiones generales: 1) aun cuando son obervables ciertas modificaciones en la interpretación y definición de la inteligencia éstas tienden a agruparse en dos ejes: la herencia biológica y el determinismo sociológico. Lo que denominan como preeminencia de la «ideología del dotado», 2) dentro de esta última concepción hay un prototipo de la persona inteligente «cibernético» de altos procesos cognitivos y pensamiento abstracto homologable a la computadora. 3) la inteligencia posee una alta valoración social y, 4) La Identidad Social es un factor organizador importante de las argumentaciones según el grado de implicación que se tenga con el objeto.

En trabajos posteriores, Carugati (Carugati, 1997; Carugati \& Selleri, 1998) ha seguido trabajando en la relación entre conocimiento «científico»e «ingenuo» de la Inteligencia encontrando que lo que los expertos dicen a los padres sigue centrándose en el desarrollo biológico del niño y en los contextos sociales y cambios demográficos. El dilema herencia vs. ambiente (natura-nurture) se mantiene como elemento clave de las argumentaciones. De esta forma, el conocimiento ingenuo también tiene una variedad de visiones sobre el desarrollo que no hace otra cosa que reflejar lo que sucede en el ámbito científico. Hay una información social que se difunde y comunica que tiene tres cualidades fundamentales: es social porque se comparte y proviene de otros; a menudo conlleva una demanda de aprendizaje y, no presenta una forma simple o singular sino variada y contradictoria. Subyace a esto una presión social que lleva a adquirir ítemes particulares de conocimiento.

Lo que se intenta remarcar es que el conocimiento de los expertos es selectivamente difuso y cambia a lo largo del tiempo tanto en contenido como en el énfasis dado a algunos aspectos sobre otros. Como contraparte, los sujetos deben afrontar y manejar esta pluralidad creando tipos de racionalidad que implican la polifasía cognitiva, las personas comunes hacen lo mismo que muchos o todos los expertos.

Hay temas que aparecen como poco familiares o con forma de «puzzle» con los que debemos familiarizarnos a fin de ser capaces de tomar responsabilidades y decisiones respecto de él. Como indican Carugati \& Selleri (1998), las representaciones sociales son ante todo el procesamiento de la información de un estímulo social extraño y, principios organizadores de ideas, prototipos normativos, y prácticas sociales que conforman los productos borrascosos de la sociedad que piensa.

De esta forma, hay dos puntos importantes que condicionan las argumentaciones respecto de las diferencias de inteligencia entre los individuos: 1) la no-familiaridad con argumentaciones respecto del objeto de representación, unida a la mayor o menor explicabilidad o falta de información que se vivencia respecto de aquél y, 2) el principio de identidad, para algunas categorías sociales (como maestros o padres) la inteligencia 
es una parte relevante de las experiencias cotidianas y, por lo tanto, elemento importante de sus identidades.

Mugny \& Carugati (1989) encontraron que las personas que subjetivamente no encuentran un modelo explicativo alternativo a su disposición y vivencian una ausencia de información, elegían como referencia a la teoría de desigualdad natural. Describían la inteligencia en términos de «don» y, responsabilizaban a la competencia de los maestros pero como garantes de una inteligencia de «menor categoría» referida a incorporar normas y reglas sociales. En este mismo sentido, la falta de información disponible lleva a confiar en disciplinas como la biología y la matemática como medios para dar sentido a las diferencias individuales. La biología puede ser vista (desde una óptica académica) como la causa de las habilidades humanas mientras que las matemáticas pueden tomarse como el «termómetro» de esas habilidades: en la creatividad del pensamiento social, las personas combinan ambas disciplinas en una relación causal donde los efectos juegan también el rol de causas.

De la mano de elementos fundamentales que se desprenden de los orígenes cognitivos y funciones de las representaciones sociales sugeridas por Moscovici, la transformación de algo no familiar en familiar, la relación entre representaciones sociales e identidad social y, sobre la base de la investigación de Mugny \& Carugati (1989), nos propusimos llevar a cabo esta investigación. Como objetivo general nos planteamos responder a la hipótesis general de la existencia de un solapamiento entre las teorías explícitas e implícitas de la inteligencia. Es decir, esperamos que aparezcan en nuestra muestra diversidad de dimensiones, tópicos y complejidad que presenta el discurso actual de los expertos. De esta proposición general, deducimos otros dos enunciados hipotéticos: 1) aquellos que vivencian el tema como más inexplicable e irresoluble apelarán más a argumentaciones y prototipos cercanos a la desigualdad natural o el «dotado» y, 2) allí donde el rol social se vea más implicado serán más salientes argumentaciones que protegan la identidad social cuestionada.

\section{Método}

\section{Muestra}

Un total de 286 sujetos respondieron el cuestionario administrado. Los sexos se distribuyen de manera relativamente proporcional (59\% Mujeres y 39\% Hombres). La media de edad es de 38 años (DS: 10,79). Respecto de la ocupación, 206 sujetos trabajan en el área educativa mientras que los 80 restantes se dedican a otras actividades.

\section{Instrumento y Procedimiento}

Los sujetos respondieron a un cuestionario autoadministrado compuesto por: 1) una escala integrada básicamente por los ítemes del Cuestionario 1 (Inteligencia) de la investigación de Mugny \& Carugati (1989); 2) una escala sobre Características de la Persona Inteligente y Datos Sociodemográficos como sexo, edad y ocupación. 
En relación a los ítemes extraídos de la investigación de Mugny \& Carugati (1989) fueron seleccionados utilizando el criterio de saturación factorial mayor o igual que .40, quedando constituida por un total de 43 ítemes. Para la construcción de la escala de prototipo de la persona inteligente, se realizó una etapa pre-test con técnica de asociación libre. Del análisis de este último se extrajeron treinta características prototípicas.

Las escalas son de tipo Likert con un continuo de respuesta de 1 (totalmente en desacuerdo) a 7 (totalmente de acuerdo). Se calcularon para las escalas los índices de fiabilidad interna medidos por el Alpha de Cronbach que arrojaron puntuaciones satisfactorias: 0.89 para la escala de definición de la inteligencia y 0.90 para la escala de prototipo.

En este trabajo presentamos los análisis correspondientes a la escala Definiciones de la Inteligencia y a la escala Prototipo de la persona inteligente y sus asociaciones con algunas variables predictoras.

\section{Resultados}

\section{Definiciones y Orígenes de la inteligencia}

Los ítems que evaluaban la subdimensión sobre los orígenes y definiciones de la inteligencia ( 1 al 43), fueron sometidos a una análisis factorial, la solución final después de la rotación varimax arroja diez factores que explican el 56\% de la varianza.

\section{Tabla 1: Estructura Factorial Dimensión “Orígen y Definición de la Inteligencia"}

\begin{tabular}{l|l|l|l|l|l|l|l|l|l|l}
\hline & F1 & F2 & F3 & F4 & F5 & F6 & F7 & F8 & F9 & F10 \\
\hline Adaptativa (9,2\%) & & & & & & & & & & \\
\hline 31: saber cuidarse uno mismo & .81 & & & & & & & & & \\
\hline 30: adaptarse ambiente físico & .80 & & & & & & & & & \\
\hline 18: idem 31 & .65 & & & & & & & & & \\
\hline 29: adaptarse a sociedad & .64 & & & & & & & & & \\
\hline 32: sacar ventajas oportunidades & .64 & & & & & & & & & \\
\hline 16: presentarse uno mismo & .62 & & & & & & & & & \\
\hline Social y Personalidad (8,8\%) & & & & & & & & & & \\
\hline 17: tener buenas maneras & & .78 & & & & & & & & \\
\hline 15: adaptarse a la escuela & & .77 & & & & & & & & \\
\hline 14: adaptarse a ideología dominante & & .74 & & & & & & & & \\
\hline 41: problema de personalidad & & .53 & & & & & & & & \\
\hline
\end{tabular}


Psicodebate. Psicología, Cultura y Sociedad

\begin{tabular}{|c|c|c|c|c|c|c|c|c|c|c|}
\hline & $\mathbf{F 1}$ & $\mathbf{F 2}$ & F3 & F4 & F5 & F6 & $\mathbf{F 7}$ & $\mathbf{F 8}$ & F9 & $\mathbf{F 1 0}$ \\
\hline 13: conforme normas sociedad & & .52 & & & & & & & & \\
\hline 40: cuestión de carácter & & .50 & & & & & & & & \\
\hline 26: computadora es el modelo & & .43 & & & & & & & & \\
\hline \multicolumn{11}{|l|}{ Ideológica $(7,8 \%)$} \\
\hline 22: ricos para justificar su poder & & & .80 & & & & & & & \\
\hline 25: adaptarse ademandas econ. y tecnológicas & & & .69 & & & & & & & \\
\hline 21: hombres definen a conveniencia & & & .68 & & & & & & & \\
\hline 24: a través I se reafirma la injusticia social & & & .64 & & & & & & & \\
\hline 38: escuela crea diferencias intelectuales & & & .57 & & & & & & & \\
\hline 23: test de inteligencia sesgados. & & & .52 & & & & & & & \\
\hline \multicolumn{11}{|l|}{ “Don” y Logro Académico $(5,57 \%)$} \\
\hline 05: logica y matematicas & & & & .66 & & & & & & \\
\hline 04: vaya bien en la escuela & & & & .58 & & & & & & \\
\hline 08: capacidad pens.abstracto & & & & .58 & & & & & & \\
\hline 03: entender al maestro & & & & .46 & & & & & & \\
\hline 07: currícula media empobrece & & & & .43 & & & & & & \\
\hline 09: act.rigurosa pensam.y accion & & & & .36 & & & & & & \\
\hline 37: escuela revela diferencias ya existentes & & & & .34 & & & & & & \\
\hline 39: potenc.aptitudes tarea educ. & & & & .33 & & & & & & \\
\hline \multicolumn{11}{|l|}{ Sociológica $(4,96 \%)$} \\
\hline 19: padres valorizan & & & & & .72 & & & & & \\
\hline 12: entrenamiento y practica & & & & & .60 & & & & & \\
\hline $\begin{array}{l}\text { 11:I aparece si hay oportunidades para su } \\
\text { desarrollo y concreción }\end{array}$ & & & & & .58 & & & & & \\
\hline 20: cada cultura tiene su propia definición de la I & & & & & .44 & & & & & \\
\hline \multicolumn{11}{|l|}{ Disfunción Rol Pedagógico $(4,83 \%)$} \\
\hline 43: fracaso evitable c/más paciencia de maestros & & & & & & .76 & & & & \\
\hline 42: fracaso por falta comprensión de los maestros & & & & & & .75 & & & & \\
\hline 36: escuela acentúa diferencias & & & & & & .45 & & & & \\
\hline
\end{tabular}




\begin{tabular}{l|l|l|l|l|l|l|l|l|l|l}
\hline & F1 & F2 & F3 & F4 & F5 & F6 & F7 & F8 & F9 & F10 \\
\hline Capital Cultural (4,26\%) & & & & & & & & & & \\
\hline 34: chicos I provienen de padres que valoran la I & & & & & & & .67 & & & \\
\hline 33: de tal padre tal hijo & & & & & & & .64 & & & \\
\hline 35: Dime ocupación de padres y diré I del niño & & & & & & & .63 & & & \\
\hline Científica (4,21\%) & & & & & & & & & & \\
\hline 27: sólo ciencia define I & & & & & & & & .68 & & \\
\hline 28: test inteligencia miden con precisión & & & & & & & & .62 & & \\
\hline 10: Cerebro es el lugar donde nace la I & & & & & & & & .46 & & \\
\hline Diferencial (3,56\%) & & & & & & & & & \\
\hline 02: Currícula más avanzada para los alumnos I & & & & & & & & & .62 & \\
\hline 06: tareas relacionan padres con escuela & & & & & & & & & .51 & \\
\hline Irresolubilidad (3,27\%) & & & & & & & & & \\
\hline 01: Diferencias de I entre los individuos es un \\
problema que la ciencia no puede resolver
\end{tabular}

El factor uno, que explica el 9,2\% de la varianza, agrupa a los ítems 31: "ser inteligente significa saber cómo cuidarse uno en la vida" (.81); 32: "Ser inteligente significa saber cómo sacar ventajas de las oportunidades" (.65); 30: "La inteligencia define la adaptación del individuo a su ambiente físico" (.64); 29: "Inteligencia es la capacidad individual de adaptarse a la sociedad en que uno vive" (.64); 16: "Ser Inteligente significa saber cómo presentarse a uno mismo de la mejor manera" (.62). La mayoría de los ítems enfatizan aspectos funcionales de la inteligencia, siendo los principales: la adaptación y acomodación al ambiente tanto físico, interpersonal, educacional y más ampliamente social. Es similar en su contenido a la Competencia Social de Sternberg ahora denominada como exitosa a la que subyace un buen intercambio individuo-ambiente. Denominamos a este factor como Definición Adaptativa.

El segundo factor explica el $8,8 \%$ de la varianza y agrupa a los ítems: $13:$ "Ser inteligente significa estar conforme con las normas de la sociedad que se ha vuelto burocrática" (.78); 40: "La inteligencia es sobre todo una cuestión de carácter" (.77); 41: "La inteligencia es un problema de personalidad" (.74); 15: "Ser inteligente significa saber adaptarse a la escuela (.53); 14: "Una persona inteligente es alguien que se puede adaptar a la ideología dominante" (.52); 17: "Ser inteligente significa tener «buenas maneras», estar «bien educado» (.50); 26: "La computadora es el modelo perfecto de lo que es la inteligencia" (.43). Los ítems aquí parecen aludir a aspectos dirigidos a la conformidad con las reglas sociales, se otorga un valor positivo a esta conformidad y se subsume a la personalidad y el carácter (iteems 40-41), probablemente como la parte 
de la persona que debe ser moldeada según las normas societales. Esta definición, pone a la escuela como termómetro y, por lo que parece, a la computadora como modelo. Esto nos hace reflexionar sobre una inteligencia de segunda categoría, ante lo que no nos da la naturaleza, podemos potenciar nuestro carácter y personalidad hacia atributos adaptativos y sociales, y esa es la función de la escuela. Definimos a este factor como Definición Social y Personalidad.

El tercer factor explica el 7,8\% de la varianza y está integrado por los ítemes: 22: "Los ricos inventaron la noción de inteligencia para justificar su riqueza y poder"(.80); 24: "A través de la Inteligencia se reafirma la desigualdad social, cuando aparece, es el rico quien la posee" (.69); 25: "La inteligencia es un invento de nuestra sociedad con el propósito de adaptarse a las nuevas demandas económicas y a las actuales condiciones tecnológicas" (.68); 23: "Los test de inteligencia son sesgados: todo lo que hacen es medir las diferencias (particularmente económicas) que existen entre las diferentes categorías sociales" (.64); 21: "Si los hombres son vistos como más inteligentes que las mujeres, es porque los hombres definen la inteligencia a su conveniencia" (.57); 38: "Es la escuela la que crea diferencias intelectuales entre los individuos" (.52). Aquí, a la hora de definir la inteligencia, se da saliencia a una postura relativista pero un relativismo que proviene de relaciones de dominación y asimetría. De grupos hegemónicos que tienen en su poder la implantación de modelos sociales. La presencia de ítemes que hablan de la inteligencia y la injusticia social y su uso y manipulación por parte de unos grupos sobre otros (ricos sobre pobres, hombres sobre mujeres), nos lleva a denominar esta dimensión como Definición Ideológica.

El cuarto factor explica el 5,57\% de la varianza y lo conforman los ítemes: 05: "La lógica y las matemáticas son los prototipos de la inteligencia" (.66); 04: "Tienes que ser inteligente para que te vaya bien en la escuela" (.58); 08: "La inteligencia tiene que ver con la capacidad de pensamiento abstracto" (.58); 03: "Inteligencia es la capacidad del alumno para entender al maestro" (.46); 07: "Basar la currícula en la inteligencia media del alumno da como resultado el empobrecimiento de los más inteligentes" (.43); 37: "La escuela sólo revela las diferencias de inteligencia que ya existen en virtud de diferentes antecedentes (bagajes) sociales" (.36); 39: "El potenciar aptitudes debería ser el objetivo central de la tarea educativa (.34); 09: "Inteligencia es antes que nada una actitud rigurosa en pensamiento y acción" (.33). Aquí es el logro académico lo que se enfatiza, es decir, una definición dada por el contexto educativo. Sin embargo también están presentes ítemes cercanos a una concepción en términos de altos procesos cognitivos (08: capacidad de pensamiento abstracto, 05: lógica y matemática, 09: actitud rigurosa en pensamiento y acción); y que reflejan una concepción elitista subyacente (07: inteligencia media empobrece currícula). Ante una postura cercana a la desigualdad natural, el poder de la escuela se relativiza y se acrecienta el del contexto familiar (item 37). Hay aquí, a nuestro criterio, indicios de la doble arbitrariedad de la tarea educativa que plantea Bourdieu y su relación con el capital cultural pero, en términos positivos. Del mismo modo, aquí el logro académico se evalúa en términos de altos procesos cognitivos. Denominamos a esta dimensión como de Definición del «don» y logro académico. 
En el factor cinco, que explica el 4,96\% encontramos los ítemes: 12: "La inteligencia no aparece si no hay oportunidades para su desarrollo y concreción" (.72); 19: "Si los padres no valoran un determinado aspecto, el esfuerzo por enseñarlo será en vano" (.60); 11: "El entrenamiento y la práctica son la base de la inteligencia" (.58); 20: "Cada cultura tiene su propia definición de la inteligencia" (.44); En este factor se observan a las prácticas y el entrenamiento como medios a través de los cuales la inteligencia se desarrolla. A diferencia del factor anterior, aquí se refuerza una concepción más sociológica y contextual. Los ítemes 11 y 12 aluden a la escuela, el item 19 incorpora la valorización de los padres y el 20 nos refiere a una concepción de la Inteligencia más relativa e "independiente" de la naturaleza o biología. Son los contextos familiares y educativos los que garantizan (u obstaculizan) el desarrollo de la inteligencia. En términos de Bordieu, la escuela tiene un rol que cumplir, su función remedial está allí cuando no haya padres que valoricen o tengan recursos para nutrirse. En oposición al factor 4 que negaba en última instancia la posibilidad de intervenir ante un fenómeno que aparece como «dado» (natural), denominamos a esta dimensión como Definición Sociológica, ya que, se pone énfasis en la escuela y la familia como agentes de cambio.

El factor seis explica el 4,83\% de la varianza y agrupa a los ítemes: 43: "El fracaso podría, en general, evitarse si los maestros tuvieran más paciencia" (.76); 42: "El fracaso escolar generalmente tiene que ver con la falta de comprensión o entendimiento por parte del maestro" (.75); 36: "La escuela acentúa las diferencias de inteligencia que existe entre los individuos" (.45). Aquí se reconoce la función que el ámbito educativo tiene en el desarrollo de la inteligencia pero desde una mirada crítica ya que, los maestros fallan en su rol pedagógico que es el recurso necesario para hacer aparecer la inteligencia. Esta definición puede denominarse Definición en términos de disfuncionalidad del rol pedagógico.

El factor siete explica el 4,26\% de la varianza y queda conformado por los ítemes: 34 : "Los chicos inteligentes provienen de familias en las que los padres valoran la inteligencia" (.67); 33: «De tal padre tal hijo» esto es igualmente cierto para la inteligencia" (.64); 35: "Dime la ocupación de los padres y te diré la inteligencia del niño" (.63); Aquí son las diferencias entre los individuos como desigualdades sociológicas pre-existentes lo que se enfatiza. Esta subdimensión refiere claramente, a nuestro criterio, al Capital Cultural. La Inteligencia aparece mediatizada, en gran medida, por la familia, el estatus social y ocupacional

El factor ocho explica el 4,21\% de la varianza y subsume a los ítemes: 28 : "Los test de inteligencia permiten medir con precisión las capacidades intelectuales de los alumnos" (.68); 27: "Sólo la ciencia puede definir lo que la inteligencia es" (.62); 10: "El cerebro es el lugar donde nace la inteligencia" (.46). Consideramos a este factor como un aspecto complementario del 4 , pues estaría más cercana a una definición que reafirma desigualdad natural (item 10) pero en el que se otorga a la ciencia y sus producciones (test) la autoridad de definir lo que la inteligencia es. Podemos denominarla, Definición Científica.

En el factor nueve encontramos a los ítemes 02: "debería haber una currícula más avanzada para los alumnos inteligentes" (.62) y 06: "Las tareas o deberes son importantes porque posibilitan que se establezca una relación entre los padres y la escuela" (.51). En 
Psicodebate. Psicología, Cultura y Sociedad

alguna medida completa la idea del factor 4 y 8 , pues alude más a una concepción de inteligencia como «don» aunque, a diferencia del factor 8 , aquí el énfasis no está puesto en la autoridad científica sino en resolver en el ámbito educativo el problema de la desigualdad. Ante inteligencias desiguales se propone una pedagogía diferencial. Los inteligentes deben tener una currícula más avanzada y que la escuela y los padres son los agentes más implicados en su seguimiento. Podemos pensar en ésta como en una Definición Diferencial de la inteligencia.

El factor diez está formado sólo por el ítem 01: "la existencia de diferencias de inteligencia entre los individuos es un problema misterioso que la ciencia no puede resolver" (.65). Puede dar fuerza a la idea de que creer en las diferencias de inteligencia como problema resoluble o irresoluble es lo que condiciona cualquier razonamiento posterior. Llamamos a este factor como Irresolubilidad.

A partir de esta solución factorial, podemos brevemente señalar la importancia de las dimensiones más sociológicas a la hora de definir la Inteligencia lo cual enfatizaría por un lado la concepción de la Inteligencia como una construcción social y, por el otro, la presencia de una definición funcional hegemónica que responde a las necesidades societales actuales.

Sin embargo, tampoco podemos excluir las dimensiones que apuntan a la idea de una desigualdad natural. Su presencia, aunque más moderada en términos de la varianza explicada por los factores que las representan, aún es fuerte y actualiza en el debate la dicotomía «natura-nuture» $\mathrm{o}$ «determinismo biológico vs. determinismo sociológico».

\section{El prototipo de la persona inteligente}

Las treinta características que forman la Escala de Imagen de la Persona Inteligente fueron sometidas a un análisis factorial. Un estudio cuidadoso de las soluciones factoriales nos indicó que la solución de cuatro factores era la más interpretable. Una vez realizada la rotación varimax, la estructura impuesta explica el $48 \%$ de la varianza.

\section{Tabla 2: Estructura Factorial: Prototipo Persona Inteligente}

\begin{tabular}{l|c|c|c|c}
\hline & F1 & F2 & F3 & F4 \\
\hline Individualista (18\%) & & & & \\
\hline 28: Poco Sociable & .80 & & & \\
\hline 19: Solitaria & .75 & & & \\
\hline 07: Egocéntrica & .73 & & & \\
\hline 15: Agresiva & .71 & & & \\
\hline 10: Soberbia & .70 & & & \\
\hline 18: Dominante & .69 & & & \\
\hline
\end{tabular}


Representaciones Sociales de la Inteligencia

\begin{tabular}{|c|c|c|c|c|}
\hline & F1 & $\mathbf{F} 2$ & F3 & F4 \\
\hline 27: Padres Sin dinero & .66 & & & \\
\hline 04: Fría & .63 & & & \\
\hline 12: Individualista & .56 & & & \\
\hline 17: De Flia. Intelectual & .55 & & & \\
\hline 09: Superior & .44 & & & \\
\hline \multicolumn{5}{|c|}{ Valorado Socialmente $(12 \%)$} \\
\hline 29: Con futuro & & .76 & & \\
\hline 30: Valorado & & .73 & & \\
\hline 22: Buenas oportunidades & & .71 & & \\
\hline 26: Con buena escuela & & .70 & & \\
\hline 11: Reconocida socialmente & & .53 & & \\
\hline 21: Constante & & .45 & & \\
\hline 24: Asertiva & & .40 & & \\
\hline \multicolumn{5}{|l|}{ Sociable / Emocional (11\%) } \\
\hline 03: Tolerante & & & .73 & \\
\hline 06: Sociable & & & .67 & \\
\hline 14: Abierta & & & .65 & \\
\hline 25: Empática & & & .58 & \\
\hline 23: Emocional & & & .55 & \\
\hline 08: Simpática & & & .53 & \\
\hline 01: Capac. Adaptación & & & .38 & \\
\hline 02: Trabajadora & & & .34 & \\
\hline \multicolumn{5}{|l|}{ Tradicional (5\%) } \\
\hline 16: Reflexiva & & & & .75 \\
\hline 20: Capac. de Abstracción & & & & .68 \\
\hline 05: Creativa & & & & .50 \\
\hline 13: Lista & & & & .44 \\
\hline
\end{tabular}

En el primer factor, que explica el $18 \%$ de la varianza encontramos contribuyendo a características tales como: Soberbia (.80), Egocéntrica (.75), Individualista (.73), Solitaria (.71), Dominante (.70), Agresiva (.69), Superior (.66), Poco Sociable (.63), Padres sin dinero (.56), Fría (.55), De Familia Intelectual (.44). A nuestro criterio, se refleja aquí un 
protipo de persona carente de habilidades sociales, individualista y, más centrado en sí mismo. Se enfatiza la idea de alguien superior y hay una completa ausencia de emocionalidad. Podemos definir a este perfil como Individualista.

El segundo factor explica el $12 \%$ de la varianza y en él encontramos a los ítemes: Buenas Oportunidades (.76), Valorado (.73), Con buena escuela (.71), Con Futuro (.70), Constante (.53), Reconocida Socialmente (.45) y Asertiva (.40). Claramente se agrupan aquí lo que en una de nuestras hipótesis planteábamos. A saber, que independientemente de la diferencia en el énfasis discursivo respecto de ella, la inteligencia es reconocida como un valor social. Describir a una persona como inteligente no sólo permite evaluarlo y clasificarlo sino que tiene un papel fundamental en la posición social y privilegios asociados a los que la persona así clasificada puede anhelar y obtener. Definimos a este perfil como Reconocimiento Social.

El tercer factor explica el $11 \%$ de la varianza y nos reúne a: Tolerante (.73), Sociable (.67), Trabajadora (.65), Simpática (.58), Emocional (.55), Abierta (.53), Empática (.38) y Capacidad de adaptación (.34). A diferencia del factor uno, aquí encontramos juntas a características que describen el perfil de una persona inteligente como alguien que tiene recursos de sociabilidad y emoción. El aspecto comunicativo, las actitudes pro-sociales y las habilidades sociales nos presentan un prototipo que denominamos como el Sociable y Emocional.

Por último, en el cuarto factor que nos explica un 5\% de la varianza, aparecen: Capacidad de Abstracción (.75), Reflexiva (.68), Creativa (.50) y Lista (.44). Este conjunto de ítemes nos recuerda a la caracterización de la persona inteligente como aquella que destaca por ser «brillante», ya sea por su alta capacidad cognitiva, por su capacidad de reflexión, su creatividad o nivel de astucia. En este sentido, estas características parecen como más híbridas y responden al modelo más frecuente y tradicional del inteligente, el que "sobresale" independientemente del campo o área en que lo haga (Filósofo como Kant en su reflexión, Científico como Einstein, por su trabajo con entidades abstractas o, alguien listo como Onassis o creativo como Bethoven o un Mozart). Nos parece pertinente denominar a este perfil como Tradicional.

De esos perfiles prototípicos podemos indicar que están presentes dimensiones que, por ausencia o por presencia, son importantes a la hora de definir la inteligencia. Por un lado, se resaltan los atributos sociales que apuntan a una buena relación del individuo con su ambiente en términos de habilidades de relación y adaptación. Por el otro, aparece la emocionalidad y la empatía, aspectos que no estaban muy presentes en la investigación de Mugny \& Carugati (1989) y que nosotros ya obtuviéramos en la etapa pre-test. Esto también se relaciona seguramente con el énfasis que en los últimos años se observa en los discursos de algunos expertos de nutrir al término Inteligencia de atributos emocionales.

La presencia de una dimensión que refuerza la valoración social de la inteligencia confirma nuestra idea de los importantes privilegios a ella asociados. Por último, y de manera congruente con lo que mencionábamos acerca de las dimensiones de las definiciones de la Inteligencia, nos aparece un perfil, el tradicional, que apunta más a una concepción de dotado, a algo con lo que se nace y con lo que se sobresale. 


\section{La Resolución del Conflicto Sociocognitivo}

Con el fin de evaluar la hipótesis de la no-familiaridad como principio organizador de una determinada representación social y, siguiendo la estrategia utilizada por Carugati \& Mugny (1989), los sujetos fueron reagrupados según sus respuestas al item: "La existencia de diferencias de inteligencias entre los individuos es un problema misterioso que la ciencia no puede resolver". La afirmación refiere a dos elementos componentes de la no-familiaridad: 1) La existencia de la no-familiaridad se asegura por la inhabilidad de las ciencias (en tanto que altas fuentes de autoridad) de proveer de una explicación racional al fenómeno; y 2) Lo que es no-familiar se define en términos de que la existencia de diferencias entre los individuos es algo para lo cual no hay ninguna explicación exhaustiva o definitiva, aún en los círculos científicos.

Aquellos sujetos que en su respuesta marcaron 1,2,3 son aquellos para quienes «la inteligencia no es un problema irresoluble», y por tanto mantienen con el objeto lo que denominamos como relación de «Familiaridad». Los sujetos que en su respuesta marcaron 5, 6, 7, son aquellos para quienes «las diferencias de inteligencia entre los individuos es algo extrañamente problemático y sin solución, aun científicamente». Estos sujetos mantienen con el objeto una relación de «No Familiaridad».

Una vez obtenida esta agrupación, y tomándola como variable independiente, procedimos a realizar un análisis de varianza sobre cada una de las soluciones fatoriales obtenidas en las escalas sobre Definición de la Inteligencia y Prototipos de la Persona Inteligente; tomando a estas últimas como variables dependientes.

\section{Tabla 3: Definiciones de la Inteligencia y Familiaridad/No Familiaridad}

\begin{tabular}{lcccc}
\hline Definiciones & Familiaridad & No Familiaridad & F & Sig. \\
\hline 1-Adaptativa & $-0,20$ & $-0,8$ & .22 & - \\
\hline 2- Social y Personalidad & $-0,36$ & 0,11 & 1,09 & - \\
\hline 3-Ideológica & $-0,13$ & 0,35 & 12,66 & .000 \\
\hline 4-Don y Logro Académico & $-0,18$ & 0,11 &, 92 & - \\
\hline 5-Sociológica & 0,25 & $-0,28$ &, 15 & - \\
\hline 6-Disfunción Rol Pedagógico & $-0,66$ & 0,16 & 2,81 & .095 \\
\hline 7-Capital Cultural & $-0,35$ & $-0,83$ &, 038 & - \\
\hline 8-Científica & 0,55 & $-0,10$ & 1,38 & - \\
\hline 9-Diferencial & $-0,12$ & 0,28 & 8,92 & .003 \\
\hline 10-Irresoluble & $-0,42$ & 0,88 & 133,8 & 000 \\
\hline
\end{tabular}

Prototipos de Personas Inteligentes y Familiaridad/No Familiaridad 
Psicodebate. Psicología, Cultura y Sociedad

\begin{tabular}{lcccc}
\hline Prototipos & Familiaridad & No Familiaridad & F & Sig. \\
\hline 1- Individualista & $-0,53$ & 0,94 & 1,167 & - \\
\hline 2- Valorado Socialmente & $-0,69$ & 0,12 & 1,963 & .16 \\
\hline 3- Sociable y Emocional & 0,16 & $-0,50$ &, 22 & - \\
\hline 4- Tradicional & 0,64 & $-0,22$ & 4,38 & .03 \\
\hline
\end{tabular}

Respecto de los grupos definidos como Familiares y No familiares, encontramos diferencias significativas en las dimensiones Ideológica, Diferencial, de Conflicto irresoluble y tendencial en la de disfunción del rol pedagógico.

Los datos de la tabla nos muestran que los individuos del grupo de «no familiaridad», en comparación con los de «familiaridad», priorizan en la definición de la inteligencia una argumentación ideológica y enfatizan la crítica al no cumplimiento de su rol por parte de los maestros. Del mismo modo, son aquellos que se sienten menos familiares con el objeto quienes muestran una mayor tendencia a apoyar definiciones con indicadores de una desigualdad natural subyacente. Están más de acuerdo con una Pedagogía Diferencial, es decir, con una currícula especial que se adapte a una realidad incuestionable e irreversible, el que unas personas son más inteligentes que otras.

Podríamos decir que la mayor distancia que se experimenta respecto del objeto que se intenta hacer accesible, incrementa las posibilidades de explicaciones más simples u ortodoxas y disminuye la riqueza o complejidad argumentativa. En nuestro caso es la argumentación ideológica la que los no familiarizados con la problemática utilizan para dar sentido a las diferencias.

Respecto de las dimensiones del prototipo de persona inteligente, encontramos que las diferencias entre los grupos aparecen en el perfil tradicional y, aunque tendencial, en la valoración social que el ser inteligente adjudica. Aquellos que se expiden como más familiarizados apoyan un prototipo tradicional mientras que los no familiarizados reconocen más la importancia que la sociedad otorga a una persona que demuestra tener cualidades inteligentes. De alguna manera estos datos son coherentes; por un lado, quienes consideran a las diferencias de la inteligencia como un problema con solución y por lo tanto más familiar, responden según el perfil más acorde a las soluciones estándares o hegemónicas, en este caso, un modelo libre de contexto y emoción en el que sólo se enfatiza a la persona que sobresale, a quien es reflexivo, tiene capacidad de abstracción y es creativo. Por otro lado, quienes viven el problema como más irresoluble y poco familiar, sólo reafirman la alta valoración que la sociedad otorga a quien se etiqueta como inteligente independientemente de los atributos que sean necesarios para tal calificación.

\section{El principio de Identidad}

Con el fin de indagar si encontrábamos argumentaciones diferenciales según el rol social de las personas, reagrupamos a nuestra muestra entre quienes trabajan en el ámbito educativo y quienes tienen ocupaciones totalmente diferentes y sin ninguna 
relación con la enseñanza. Una vez obtenida la reagrupación, se realizó con ésta un análisis de varianza sobre cada una de las soluciones factoriales de la escala sobre Definiciones de la Inteligencia y de la escala sobre Prototipos de la Persona Inteligente.

\section{Tabla 4: Definiciones de la Inteligencia entre Maestros y No Maestros}

\begin{tabular}{lcccc}
\hline Definiciones & $\begin{array}{c}\text { Maestros } \\
(\mathbf{N = ~ 2 0 6 )}\end{array}$ & $\begin{array}{c}\text { No Maestros } \\
\mathbf{( N = \mathbf { 8 0 } )}\end{array}$ & $\mathbf{F}$ & Sig. \\
\hline 1-Adaptativa & 0,50 & $-0,13$ & 1,89 & - \\
\hline 2- Social y Personalidad & 0,29 & $-0,75$ & .62 & - \\
\hline 3-Ideológica & $-0,10$ & 0,27 & 8,37 & .004 \\
\hline 4-Don y Logro Académico & 0,42 & $-0,10$ & 1,32 & - \\
\hline 5-Sociológica & 0,77 & $-0,19$ & .044 & - \\
\hline 6-Disfunción Rol Pedagógico & $-0,66$ & 0,17 & 3,33 & .069 \\
\hline 7-Capital Cultural & 0,51 & 0,12 & 1,87 & - \\
\hline 8-Científica & 0,60 & $-0,15$ & 2,67 & .10 \\
\hline 9-Diferencial & $-0,24$ & 0,62 & 4,32 & - \\
\hline 10-Irresoluble & $-0,68$ & 0,17 & 3,44 & .065 \\
\hline
\end{tabular}

Prototipos de Personas Inteligentes entre Maestros y No Maestros

\begin{tabular}{lcccc}
\hline Prototipos & $\begin{array}{c}\text { Maestros } \\
(\mathbf{N = 2 0 6})\end{array}$ & $\begin{array}{c}\text { No Maestros } \\
(\mathbf{N = \mathbf { 8 0 } )}\end{array}$ & $\mathbf{F}$ & Sig. \\
\hline 1- Individualista & $-0,73$ & 0,18 & 4,002 & .046 \\
\hline 2- Valorado Socialmente & $-0,62$ & $-0,16$ & 2,907 & .089 \\
\hline 3- Sociable y Emocional & 0,29 & $-0,75$ &, 631 & - \\
\hline 4- Tradicional & 0,92 & $-0,23$ & 6,47 & .01 \\
\hline
\end{tabular}

Respecto de las definiciones de Inteligencia, los grupos de Maestros y No Maestros muestran diferencias significativas en las dimensiones ideológica, disfunción del rol pedagógico, científica e irresolubilidad. Son los No Maestros quienes en sus argumentaciones incorporan elementos ideológicos y de crítica social, de la misma manera y, coherentemente, son quienes, dentro de esta crítica, ubican al fracaso escolar de los individuos como resultado del no cumplimiento satisfactorio de su función por parte de los agentes educativos. Por el contrario, quienes trabajan en el ámbito educativo, muestran una mayor tendencia a dejar en las manos de la ciencia, como fuente válida de autoridad, la responsabilidad de definir lo que la inteligencia es.

Estos resultados son coherentes con el postulado de la alta implicación entre representaciones sociales e identidad, las explicaciones de sentido común se construyen 
Psicodebate. Psicología, Cultura y Sociedad

en relación a los roles y funciones de los sujetos. En este sentido, los Maestros, implicados en su rol, no pueden optar por explicaciones que los ponga a ellos como agentes del fracaso o de dominación pero sí pueden hacerlo aquellos cuyas actividades están menos comprometidas con la resolución del problema de la inteligencia. Los Maestros, de manera coherente, muestran una tendencia a buscar en la ciencia el ámbito donde encontrar la respuesta.

Respecto de los prototipos, las diferencias significativas entre los grupos aparecen en los perfiles Individualista, Tradicional y de valoración social. Los Maestros están más de acuerdo con un perfil que describe a la persona inteligente en términos más bien tradicionales y en cómo el ser inteligente promueve la valoración social de la persona. A diferencia de éstos, los No Maestros están más cercanos a caracterizar a la persona inteligente en términos individualistas.

\section{Relación entre definiciones de la Inteligencia y prototipos de personas inteligentes}

Con el objetivo de indagar en las posibles relaciones entre definiciones de inteligencia y prototipo de la persona inteligente, se realizaron análisis de correlaciones de las soluciones factoriales de ambos conjuntos de ítemes.

\section{Tabla 5: Correlaciones entre Definiciones de Inteligencia y Prototipos}

\begin{tabular}{lcccc}
\hline $\begin{array}{l}\text { Origen -Definición } \\
\text { Inteligencia }\end{array}$ & $\begin{array}{c}\text { Individual } \\
\text { Sin Emoción }\end{array}$ & $\begin{array}{c}\text { Prototipos Persona Inteligente } \\
\text { Valor Social }\end{array}$ & $\begin{array}{c}\text { Sociable } \\
\text { Emocional }\end{array}$ & Tradicional \\
\hline 1-Adaptativa & $.15^{*}$ & $.12^{* *}$ & $.32 * *$ & $.19^{* *}$ \\
\hline 2-Social y Personalidad & $.22^{*}$ & $.13^{*}$ & $.15^{*}$ & $-.14^{*}$ \\
\hline 3-Ideológica & $.20^{*}$ & -.01 & .06 & $-.11^{* *}$ \\
\hline 4-Don y Logro Académico & $.16^{* *}$ & .08 & -.02 & $.26 *$ \\
\hline 5-Sociológica & $-.14^{*}$ & $.14^{* *}$ & .08 & $.09 * * *$ \\
\hline 6-Disfunción Rol pedagógico & .09 & $.13^{* *}$ & $.11 * *$ & .06 \\
\hline 7-Capital Cultural & .07 & .04 & -.07 & .06 \\
\hline 8-Científica & .21 & .04 & .06 & $.14 * *$ \\
\hline 9-Diferencial & $.12 * *$ & -.01 & -.08 & .04 \\
\hline 10-Irresolubilidad & .07 & $.21 *$ & -.06 & $-.21 *$ \\
\hline
\end{tabular}

$* * \mathrm{p}<0.001 ; * \mathrm{p}<0.05 * \mathrm{p}<0.1$

La concepción adaptativa de la inteligencia correlaciona positivamente con los cuatro prototipos; de manera parcialmente coherente, la concepción social no se 
relaciona con una caracterización más híbrida que excluye el contexto, pero sí responden a una caracterización individualista. La concepción más crítica o ideológica se relaciona más con el prototipo individualista que con el tradicional. Podemos pensar que el modelo individualista es el prototipo hegemónico y que a la hora de caracterizar, lo que se hace es describir el prototipo dominante. La definición de la inteligencia que se basa en el don y el logro académico. No sorprende que correlacione positivamente sólo con aquellos prototipos que no tienen en cuenta al medio o contexto y hagan caso omiso de la emocionalidad (cuya carencia homologábamos al modelo cibernético). La concepción sociológica, por su parte, se relaciona con un prototipo del inteligente como valorado socialmente y se inclina más por un perfil tradicional que individualista. Aunque no significativa, hay una relación positiva con la sociabilidad y emoción.

La concepción que responsabiliza a los maestros se orienta por perfiles de valoración social y sociabilidad y emoción. La concepción que enfatiza el capital cultural no muestra relación significativa con ninguno de los cuatro prototipos, aunque su relación es positiva con todos salvo con sociabilidad y emoción. De manera coherente, la definición científica, en la que subyace una concepción de desigualdad natural y que pone como fuente de autoridad a la ciencia, se relaciona con un prototipo más tradicional. Por último, una postura similar a la anterior pero que en vez de apelar a la autoridad científica se inclina por una pedagogía diferencial, se relaciona con un perfil más individualista. Estas dos últimas concepciones las consideramos como corolarios de una concepción de la inteligencia basada en la desigualdad natural, por eso aparecen relacionadas con los prototipos que excluyen tanto al medio como a la emocionalidad.

\section{Conclusiones}

En general, podemos concluir que los datos analizados aportan evidencia empírica en el sentido de las hipótesis postuladas. Tanto a la relación de la hipótesis general del solapamiento entre teorías explícitas e implícitas como a las referidas al principio de familiaridad e identidad social.

Respecto de la retroalimentación entre ciencia y sentido común, la estructura encontrada en las dimensiones sobre definiciones de la inteligencia - la varianza explicada por los distintos factores - es similar a la que describiéramos respecto de las actuales argumentaciones que tiene lugar entre los expertos. Al igual que estudios anteriores (Sternberg, 1981; Carugati,1990), vemos cómo las argumentaciones de origen científico son consumidas y transformadas para servir en la vida cotidiana o, de modo inverso, cómo la ciencia se nutre de conocimientos producidos por los miembros de un grupo de forma espontánea a través de la tradición y el consenso. Ya sea como subproducto de la ciencia o como producto de intercambios cotidianos, los sabios aficionados (Moscovici $\&$ Hewstone, 1988) definen actualmente a la inteligencia como un atributo social relacionado con la adaptación a las normas societales y explicitan argumentaciones que integran mayor número de variables contextuales. 
El sentido común refleja de alguna manera los cambios producidos en el debate científico. Las concepciones adaptativas y sociales están cerca de la renovada definición que Sternberg (1998) presenta como "inteligencia exitosa” y que apunta básicamente a un buen intercambio individuo-ambiente. En el mismo sentido, las estructuras encontradas confirman la observación de Carugati (1990) acerca del incremento de abordajes contextuales y ecológicos al momento de definir la inteligencia. Un cambio importante se está dando en los últimos años que refuerza a la inteligencia en términos de cualidades que las personas poseen o no en situaciones o contextos de aplicación específicos. Gardner (1994) enfatiza el concepto de «Inteligencias Múltiples» haciéndolo depender de las áreas o campos específicos en las que los sujetos se desempeñan.

En el mismo sentido, al igual que otros estudios empíricos recientes (Faria \& Fontaine, 1994), encontramos que la definición relativista asume un mayor peso, en comparación a Mugny \& Carugati (1989) que encontraban la desigualdad natural como la concepción más fuerte; en nuestro caso aparece más ideológica que relativa a la cultura. Sin embargo, no podemos afirmar que las concepciones más biologicistas hayan desaparecido. Tres de las dimensiones de las definiciones de la inteligencia aluden a concepciones del dotado o de desigualdad natural, la dimensión que definimos como "el dotado y el logro académico" explica una parte importante de la varianza y, otras dos dimensiones científica y diferencial - sólo se diferencian en cómo se refrenda lo que "natura provee", esto es, por medio de la ciencia y su medición psicométrica o por medio de la escuela y la programación de una currícula diferencial.

Si bien la etapa en que nuestra investigación se encuentra no nos permite enunciar afirmaciones contundentes, podemos deducir de nuestros datos la presencia de la dicotomía "determinismo biológico vs. determinismo sociológico" de la que ya hablaban Mugny \& Carugati y, por otro, la posible aparición de una inteligencia de segundo orden o nivel. Es decir, que ante algo dado o no dado por la naturaleza, queda la posibilidad de acceder a una segunda inteligencia que tiene que ver con potenciar atributos adaptativos y las habilidades sociales. Esta idea, ya planteada por Carugati (1990) es una línea interesante a profundizar.

Respecto de los perfiles de la persona inteligente, y en coherencia con lo hasta aquí mencionado, vemos que atributos emocionales y sociales se hacen más fuertes en tanto características prototípicas. Mientras en la investigación de Mugny \& Carugati (1989) éstos estaban poco contemplados en nuestro trabajo fueron deducidas de la información dada por los sujetos en la etapa pre-test. Por otro lado, también está presente un prototipo más cibernético o de altos procesos cognitivos que subyace a las definiciones que afirman una desigualdad natural. Las dimensiones que apuntan a esta última sólo correlacionan en nuestros datos con perfiles de personas inteligentes que dejan de lado tanto a los condicionantes contextuales como a las habilidades sociales (híbrido y tradicional). Por el contrario, los atributos de sociabilidad, emoción y adaptación se enfatizan, aunque de manera dispersa, en las definiciones de tinte más sociológicas.

El análisis de la hipótesis de no-familiaridad nos muestra que quienes ven a las diferencias de inteligencia como un problema sin solución (los extraños) enfatizan explicaciones ideológicas y de críticas al rol disfuncional de los maestros. Del mismo 
modo, son aquellos que se sienten menos familiares con el objeto quienes muestran mayor tendencia a apoyar afirmaciones cercanas al determinismo biológico (por ejemplo acordar con que debe haber una currícula diferencial). Ante una realidad irreversible, la escuela debe hacer diferencias. Por un lado vemos aquí como «la familiarización de lo extraño» hace prevalecer los marcos preexistentes alineando la novedad en lo ya conocido. Por otro lado, esto es coherente con lo encontrado por Mugny \& Carugati (1989) quienes observaban que la afirmación de no-familiaridad es parte de una representación muy específica en la cual, el reconocimiento de diferencias naturales se combina con una actitud discriminatoria y elitista. Al igual que estos autores, también encontramos que el efecto del ambiente que se acepta es la responsabilidad personal de los maestros la que recibe mayor énfasis por parte del grupo de menor familiaridad.

Finalmente, la mayor familiaridad se asocia a un prototipo tradicional mientras que los extraños (problema irresoluble) sólo reconocen el alto valor social que ser inteligente implica. Se reafirma aquí probablemente la fuerza que aún puede tener en el saber popular los estereotipos vinculados a la teoría o ideología del don (Doise \& Mugny, 1997).

La hipótesis de la identidad social y su función en la construcción de una determinada representación social también arroja datos interesantes. Sabemos de la función que los sistemas de creencias cumplen respecto de los posicionamientos sociales y de la complementación al interior de una representación social de funciones cognitivas (objetivación) y funciones sociales (anclaje) (Doise, Clemence \& Lorenzi-Cioldi 1993). De manera esperable entonces, quienes tienen una mayor implicación con la problemática por las funciones del rol, como es el caso de los maestros, apoyan explicaciones sociológicas que defienden su posición o ponen «las culpas en el afuera». También otorgan a la ciencia la autoridad de definir lo que la inteligencia es. Por el contrario, quienes tienen una implicación de rol mucho menor, quienes no son maestros, argumentan más en términos de crítica social (ideológicas) y señalan a los maestros como culpables del fracaso escolar de los alumnos. Esto nos hace pensar en la importancia a la hora de evaluar una representación social de las condiciones y contextos en las que surgen, las comunicaciones mediante las que circulan y las funciones a las que sirven dentro de la interacción con el mundo y con los demás (Jodelet, 1986).

Los grupos construidos por identidad social (Maestros-No Maestros) no muestran diferencias en atributos de valoración social y emoción a la hora de caracterizar el prototipo. Sin embargo, es llamativo que, los perfiles que predominan son aquellos desprovistos de condiciones ambientales o sociales, mientras los maestros lo caracterizan en términos tradicionales, los no maestros se inclinan por uno más individualista. Se reafirmaría lo ya dicho sobre la presencia del estereotipo del dotado en el saber popular.

A modo de cierre, remarcar la futura aplicabilidad de los resultados encontrados, sobre todo en el ámbito educativo. Si la Inteligencia es un concepto que además de aludir a procesos internos de procesamiento de la información incorpora a las fuerzas sociales que lo construyen (Richardson, 1994), será importante estar prevenidos de las concepciones de aquellos que en su práctica cotidiana tienen un alto nivel de implicación y responsabilidad en su desarrollo y concreción, los maestros. 
Psicodebate. Psicología, Cultura y Sociedad

\section{Bibliografía}

Agoustinos, M. \& Innes, J.M. (1990). Towards an integration of social representations and social schema theory, British Journal of Social Psychology, 29, 213-231.

Amaral, V. (1997). A Inteligência e o seu desenvolvimento: Representacoes sociais e identidades sociais. En M.B Monteiro, P. Castro (eds), Cada CabeÇa sua SentenÇa: Ideias dos adultos sobre as crianÇas. Oeiras: Celta Editora.

Bourdieu, P. \& Passeron, J.C. (1981). La Reproduccion. Barcelona, Edit. Paidós.

Carugati, F (1990). From social cognition to social representations in the study of intelligence. En G. Duveen \& B, Lloyd (De.), Social Representations and the development of knowledge. New York: Cambridge University Press.

Doise, W. \& Mugny, G. (1997). Psychologie Sociale \& développement cognitif. Paris: Armand Colin.

Doise, W. Clemence, A. \& Lorenzi-Cioldi, F. (1993). The Quantitative Analysis of Social Representations. London: Harvester Wheatsheaf.

Faria, L. \& Fontaine, A. M. (1994). Teacher's Social Representations of Intelligence: an exploratory study with Portugese Teachers. En A. Clemente, G. Musitu, E. Gutierrez (Eds.), Intervención Educativa é Desarrollo Humano (pp 29-38). Valencia: Seti Set Edic.

Flament, C. (1987). Pratiques et Représentations Sociales. En J.L Beavois, R.V. Joule, J.M. Monteil (Eds.). Théories Implicites et Conflicts Cognitifs. (pp 143-150). Cousset: DelVal.

Farr, R. (1987). Social Representations: a French Tradition of Research. Journal for the Theory of Social Behaviuor, 17, 343-369.

Fraser, C. (1994). Attitudes, Social Representations and Widespread Beliefs. Papers on Social Representations, Vol. 3, 13-25.

Gardner, H. (1984). The development of competence in culturally defined domains. En Richard A.S. \& R. A. Levine (Eds.) Culture Theory. Essays on Mind, Self and Emotions. Boston: Cambridge University Press.

Gardner, H. (1994). Multiple intelligences: The theory in practice. En Teachers College Record, Vol. 95, № 4, 557-583.

Jodelet, D. (1988). La Representación Social: Fenómenos, Concepto y Teoría. En Moscovici, S. (Ed), Psicología Social, Pensamiento y vida social. Psicología social y Problemas Sociales. Buenos Aires: Paidós.

Moscovici, S. \& Hewstone, M. (1988). De la Ciencia al Sentido Común. En Moscovici, S. (Ed), Psicología Social, Pensamiento y vida social. Psicología social y Problemas Sociales. Buenos Aires, Editorial Paidós.

Moscovici, S. (1961). Le Psychoanalyse, son image et son public. Paris: PUF. 
Moscovici, S. (1981). On Social Representations, En J.P Forgas (Ed.), Social Cognition: Perspectives in everyday understanding. Londres: Academic Press.

Moscovici, S. (1984). The phenomenon of Social Representations. En Farr, R. \& Moscovici, S. (Eds.), Social Representations. Cambridge: Cambridge University Press.

Mugny, G. \& Carugati, F. (1989); Social Representations of Intelligence. New York: Cambridge University Press.

Mugny, G. \& Doise, W. (1985). La Construcción Social de la Inteligencia. México: Trillas.

Poeschl, G. \& Doise, W. (1993). Intelligence of the self and human intelligence in general: A “superior conformity of the self” effect? En J. Piolat, M. F. Pichevin, M.C. Hurtig (Eds.), Studies on the self and social cognition. Singapur: World Scientific Publishing Co.

Richardson, K. (1994). Understanding Intelligence. Philadelphia: Open University Press.

Semin, G. R. (1987). On the relationships between representations of theories in psychology and oridinary language. En Doise, W. \& Moscovici, S. (Eds), Current Issues in European Social Psychology. Cambridge: Cambridge University Press.

Stemberg, R. \& Detterman, D. (1988). ¿Qué es la Inteligencia? Enfoque actual de su naturaleza y definición. Madrid: Pirámide.

Sternberg, R. (1985). Implicit Theories of Intelligence, Creativity and Wisdom. Journal of Personality and Social Psychology, 49, 607-27.

Sternberg, R. \& Kauffman, J. D. (1998). Human Habilities. Annual Review Psychology, 49, 479-502.

Sternberg, R.; Conway, B., Ketron, J. \& Bernstein, M.; (1981). People's conception of intelligence. Journal of Personality and Social Psychology, 49, 607-27. 
Psicodebate. Psicología, Cultura y Sociedad 